\title{
ANÁLISIS ESPECTRAL DE ONDAS SUPERFICIALES: APLICACIÓN EFECTIVA PARA LA CARACTERIZACIÓN GEOLÓGICA DE OCHO SITIOS DISTINTOS DE CIMENTACIÓN EN COSTA RICA
}

\author{
SPECTRAL ANALYSIS OF SURFACE WAVES: EFFECTIVE METHOD \\ FOR THE GEOLOGIC CHARACTERIZATION OF EIGHT DIFFERENT \\ FOUNDATION SITES IN COSTA RICA
}

\author{
Carlos A. Vargas*, Luis A. Vargas \& Mauricio T. Coto \\ MyV Soluciones Geotécnicas SA. Apartado postal: 2079-2050 San José \\ *Autor para contacto: cavargas@myv-sg.com
}

(Recibido: 24/09/09 ; aceptado: 12/12/09)

\begin{abstract}
Spectral Analysis of Surface Waves (SASW) is a geophysical technique that is spreading worldwide as a means for obtaining Shear Wave Velocity Profiles (Vs). The Vs parameter is a highly valuable aid to developing stratigraphic columns given its close correlation to the stiffness of a given geological layer. On the other hand, Vs profiles can be used to determine important parameters for Structure Engineers in the process of Foundations and Structure Design. As part of this research, nine (9) SASW tests were carried out at eight (8) different sites across Costa Rica. For each site, geological parameters were obtained: stratigraphic column based upon the Shear Wave Velocity profile, as well as geotechnical parameters: Natural Site Period (Tn), Average Vs30 (Average shear wave velocity to a depth of 30 meters) and the Site Classification as per Costa Rican Seismic Code. According to the results, the SASW profiles showed important contrasts among the different sites: site categories ranging from $\mathrm{S} 3$ to $\mathrm{S} 1$, and natural site periods ranging from 0,22 seconds to 0,61 seconds.

Keywords: Spectral Analysis of Surface Waves, Shear Wave Velocity, Natural Period, Soils, Foundations, Geophysics, Costa Rica.

Resumen: El análisis espectral de ondas superficiales es una técnica que ha extendido su uso alrededor del mundo para definir directamente la velocidad de onda cortante Vs. Esta variable es una característica muy valiosa del sustrato geológico, ya que se convierte en el esqueleto de la columna estratigráfica dibujada mediante un perfil de rigidez y correlacionada con gran precisión en las diferentes capas de la secuencia. Además, a través de este valor se derivan parámetros muy importantes para la ingeniería estructural en el proceso de cimentación de edificaciones. Los nueve sondeos efectuados, utilizando esta metodología, se realizaron en ocho sitios diferentes del país obteniendo una columna estratigráfica correlativa con la velocidad de onda (Vs), el periodo natural (Tn), el valor de Vs30 (promedio primeros 30 metros) y la clasificación del sitio de cimentación. Los ocho sitios presentan importantes variaciones en el comportamiento dinámico del sustrato clasificando desde sitios S3 hasta sitios S1 con valores de Tn que fluctúan entre 0,22 s hasta 0,61 s.

Palabras clave: Análisis espectral de ondas superficiales, velocidad de onda, periodo natural, suelos, cimentaciones, geofísica, Costa Rica.
\end{abstract}

VARGAS, C.A., VARGAS, L.A. \& COTO, M.T., 2009: Análisis espectral de ondas superficiales: aplicación efectiva para la caracterización geológica de ocho sitios distintos de cimentación en Costa Rica.- Rev. Geól. Amér. Central, 41: 37-24. 


\section{INTRODUCCIÓN}

El daño sísmico, su naturaleza y distribución, depende de la capacidad de respuesta dinámica del suelo frente a cargas cíclicas, además del adecuado diseño estructural. Esta respuesta del suelo está condicionada por múltiples factores entre los que podemos mencionar: la magnitud del sismo, los mecanismos de ruptura originales del movimiento, la clasificación, orientación y dimensión de la falla creadora del hipocentro, las características geológicas del medio, condiciones de agua subterránea, las propiedades geotécnicas del suelo-roca y la trayectoria de las ondas.

Las propiedades geotécnicas que influencian, prioritariamente, la susceptibilidad de un sustrato de suelo para la amplificación del movimiento dependen de las propiedades dinámicas, siendo el módulo de corte, $(\mathrm{G})$, y el amortiguamiento, $(\zeta)$, los principales parámetros para modelar la respuesta sísmica del suelo. Estos parámetros se relacionan con otras propiedades físicas del material, una de ellas, la velocidad de onda cortante, de la cual se derivan $\mathrm{Vs}_{30} \mathrm{y} \mathrm{T}_{\mathrm{n}}$.

En Costa Rica, la determinación de las propiedades dinámicas del suelo de manera directa, ha estado muy limitado. Los estudios realizados han sido dirigidos al análisis de la dinámica de los suelos en el Valle Central (Ramírez, 1996), principalmente, y en sectores muy específicos del gran Área Metropolitana, como estudios preliminares para la construcción de infraestructura. (p.e. el nuevo hospital de Alajuela).

Las técnicas SASW para la medición directa de la velocidad de onda en Costa Rica, se han utilizado en muy pocos estudios, específicamente dirigidos a la microzonificación sísmica del Área Metropolitana, compiladas por el Laboratorio de Ingeniería Sísmica de la UCR y realizadas por el Instituto Noruego de Geotecnia en colaboración con el Instituto Costarricense de Electricidad (Moya, et al., 2000).

\section{METODOLOGÍA}

\section{Metodología técnica}

Las ondas Rayleigh se expresan como movimientos elípticos verticales de las partículas en la superficie, en las que la dirección varia de retrograda a prograda, de un lado a otro de la elipse. Un impacto en superficie genera ondas de corte y compresión, pero la energía se dispersa como ondas Rayleigh, principalmente, en un porcentaje del $67 \%$ (Wood, 1968). Los avances tecnológicos en el sector del procesamiento de datos y la electrónica han permitido la construcción de los instrumentos necesarios con los que, ahora, se puede aprovechar este tipo de ondas, mientras que en el pasado la geofísica solo utilizaba las ondas de cuerpo (P y S) para las investigaciones, considerando a las ondas de superficie el "ruido" de desecho.

El principio fundamental del análisis de ondas superficiales (Mattews et al., 1996) se trata de que las ondas Rayleigh, viajen a una velocidad independiente de su longitud de onda en suelos uniformes y homogéneos, mientras que en sustratos estratificados donde la rigidez y la densidad son variables, la velocidad y la frecuencia se vuelven dependientes una de la otra. Para un mismo estrato, la velocidad de la onda Rayleigh es 0,919 Vs (Perez \& Izaguirre, 1982), por lo que para efectos prácticos, se convierten en lo mismo.

El análisis espectral de ondas superficiales AEOS (SASW por sus siglas en ingles) es una técnica, utilizada con mucha frecuencia alrededor del mundo, para definir la velocidad de onda cortante "in situ". El sistema SASW es un método de análisis geofísico no destructivo y no invasivo, efectuado en la superficie del terreno, en la cual se generan vibraciones sinuosoidales estacionarias con diferentes elementos de oscilación sísmica a determinadas distancias conocidas (Fig.1). Mediante un equipo procesador 


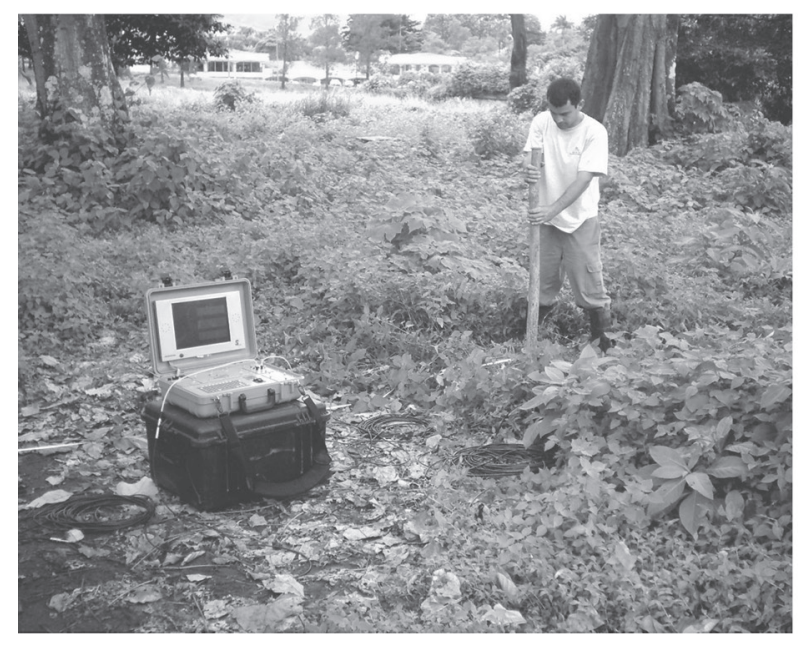

\section{Computador portátil}
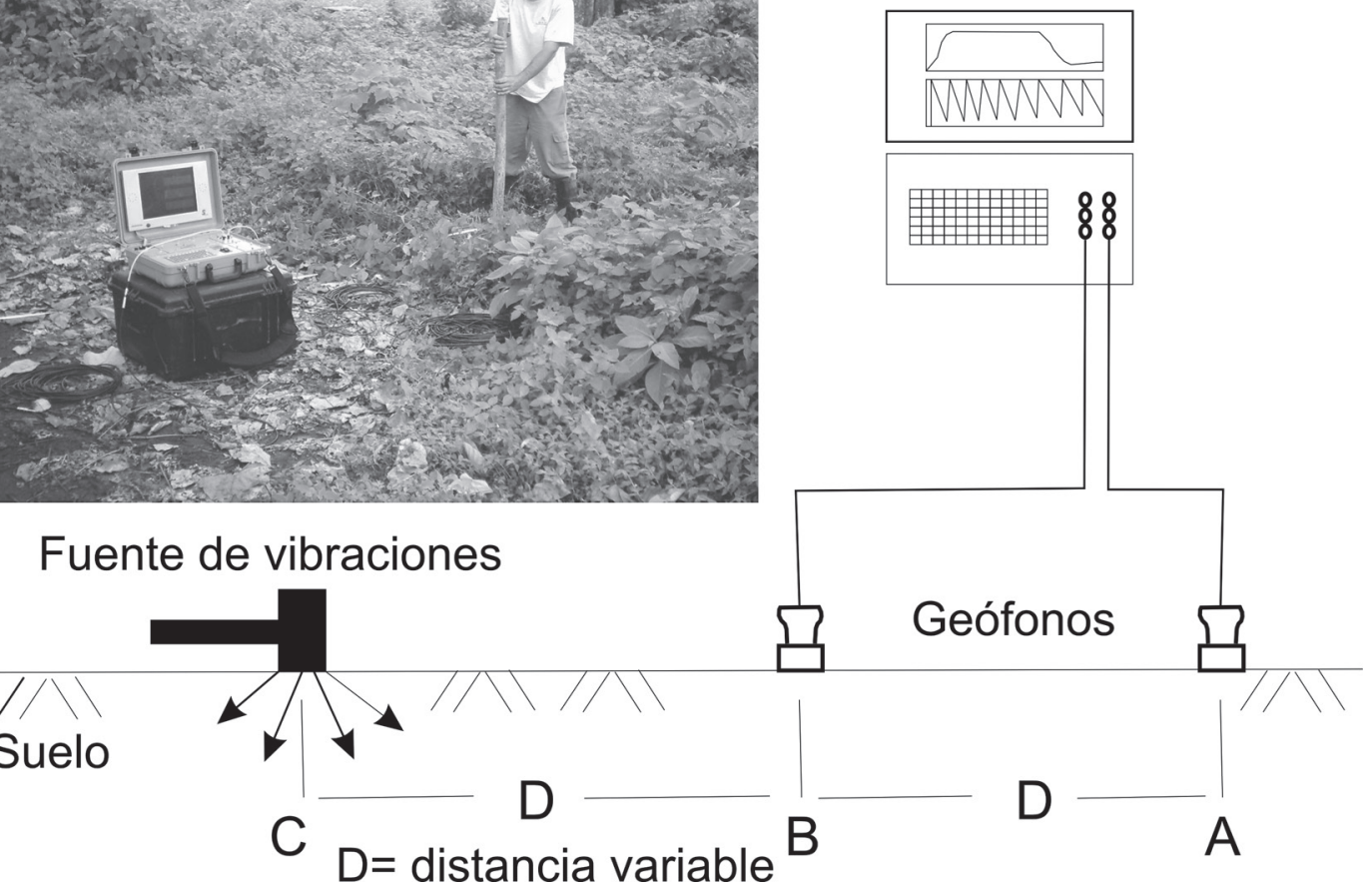

Fig. 1: Esquema de realización del ensayo SASW

electrónico digital se registran las diferentes frecuencias generadas por los elementos vibradores y se obtiene la curva de dispersión que asocia la velocidad con la frecuencia y la longitud de onda, la cual refleja las condiciones de rigidez del sitio, la estratigrafía y posibles anomalías en la compactación del material.

El dispositivo de este ensayo consiste en la instalación de dos receptores verticales separados una distancia $\Delta \mathrm{d}=\mathrm{dB}-\mathrm{dA}$ en la superficie del suelo y al lado de una fuente emisora de ruido, sobre una misma línea visual. Los datos registrados son la diferencia de fase de las ondas Rayleigh. Estos datos se transforman al dominio de frecuencias mediante la transformada rápida de Fourier, luego utilizando técnicas espectrales se calculan la transformada de Fourier de la función de correlación cruzada entre las señales seleccionadas. Tras dicha transformación, la diferencia de fase se computa para cada frecuencia. De esta forma se puede obtener el tiempo de viaje de las ondas entre los dos receptores (Kramer, 1996) como:

$$
\Delta t(f)=\frac{\phi(f)}{2 \pi f} \quad(1 \text { (Kramer, 1996) }
$$

Dado que la distancia entre los dos receptores es conocida $\Delta \mathrm{d}=\mathrm{dB}-\mathrm{dA}$ se puede calcular la velocidad y la longitud de onda como:

$$
V_{R}(f)=\frac{\Delta d}{\Delta t(f)} \quad(2 \text { Kramer, 1996) y }
$$




$$
\lambda_{R}(f)=\frac{V_{R}(f)}{f} \quad(3 \text { (Kramer, 1996) }
$$

La representación de la velocidad de las ondas superficiales Rayleigh en función de la frecuencia (o de la longitud de onda) se conoce como curva de dispersión. Se ha demostrado que la forma de las curvas de dispersión en un lugar determinado está relacionada con la variación de la velocidad de las ondas internas con la profundidad.

Los resultados obtenidos para diferentes separaciones (D) son sumados a la curva de dispersión, con la cual se representa un perfil que demuestra la velocidad de onda cortante para cada estrato, la rigidez del medio y el espesor de las diferentes capas.

La selección de las ondas Rayleigh de los demás arribos se efectúa utilizando los conceptos de fase y coherencia. La fuente vibratoria aplicada en la superficie del terreno (C) genera en los puntos A y B dos señales temporales: $\mathrm{A}(\mathrm{t})$ y $\mathrm{B}(\mathrm{t})$. Con estas señales, un Analizador de Espectros o un Ordenador calculan en tiempo real la función de coherencia, $\gamma 2 \mathrm{AB}(\mathrm{f})$, y la fase, $\theta \mathrm{AB}(\mathrm{f})$, de la función densidad espectral cruzada de energía $\mathrm{GAB}(\mathrm{f})$, en función de la frecuencia, f.

\section{Función de fase}

El concepto de función $\theta \mathrm{AB}(\mathrm{f})$ se deriva de que a cada valor de frecuencia "f" en $\mathrm{Hz}$, le corresponde un valor de desfase $\theta \mathrm{AB}(\mathrm{f})$ entre $\mathrm{A} y$ $\mathrm{B}$, estando relacionado este desfase con el tiempo de recorrido (tAB) de una onda de frecuencia "f" (Cuellar et al, 1995), entre ambos transductores mediante la expresión:

$$
t_{A B}=\frac{\theta A B(f)}{360 f} \quad \text { (Cuellar et al., 1995) }
$$

, donde $\theta \mathrm{AB}(\mathrm{f})$ viene expresado en grados, $\mathrm{f}$ en $\mathrm{Hz}$ y t $\mathrm{AB}$ en segundos. Una vez conocido tAB es inmediato determinar la velocidad de fase, VR, ya que:

$$
V_{R}=\frac{D}{t_{A B}}
$$

siendo " $\mathrm{D}$ " la distancia entre transductores (Fig. 1). La velocidad VR calculada corresponde a una longitud de onda, $\lambda$ (Cuellar et al, 1995), de valor:

$$
\lambda=\frac{V_{R}}{f}
$$

Repitiendo el proceso para diferentes frecuencias, se puede obtener la velocidad de fase "VR" en función de la frecuencia " $\mathrm{f}$ " o en función de la longitud de onda " $\lambda$ "

\section{Función de coherencia}

La función de coherencia, $\gamma 2(f)$, se utiliza para seleccionar el rango de frecuencias en las que se aplica el proceso de cálculo antes mencionado.

Esto se debe a que $\gamma 2(f)$ es una función, en el dominio de la frecuencia, análoga a la del cuadrado del coeficiente de correlación, tal que:

$$
0 \leq \gamma 2 \text { (f) } \leq 1 \quad \text { (Cuellar et al., 1995) }
$$

En esta expresión la función de coherencia valdrá 0 cuando las señales $\mathrm{A}(\mathrm{t})$ y $\mathrm{B}(\mathrm{t})$ no estén relacionadas, y valdrá 1 cuando haya una dependencia lineal entre ambas. En general, se exige un valor igual o mayor de 0,90 (Cuellar et al, 1995).

\section{Metodología experimental}

La geología de nuestro país es muy diversa, presentando variaciones importantes en las características de los materiales en distancias muy cortas, formando suelos con diferentes condiciones geotécnicas y macizos rocosos con caracterizaciones particulares.

Se han seleccionado ocho sitios distintos para el análisis SASW tanto en el Valle Central como en otras áreas del país, que varían desde zonas costeras compuestas por sedimentos aluviales inconsolidados hasta macizos rocosos sedimentarios fracturados. 
Estos sitios presentan detalles geológicos muy particulares por lo que se puede lograr una caracterización de la velocidad de onda cortante en estratos no consolidados de arenas y arcillas cuaternarias y capas soldadas y no soldadas de materiales volcánicos de edad holocena y pleistocénica hasta macizos rocosos del Terciario y Cretácico.

\section{DESCRIPCIÓN FÍSICA DE LOS SITIOS}

Los sitios seleccionados se localizan tanto en el Valle Central, la zona chorotega, el área pacifico central y el sector huetar norte (Cuadro 1), en las hojas cartográficas a escala 1:50 000, Abra, Quesada, Tilarán, Carrillo Norte, Tárcoles y Herradura (Fig. 2).

\section{Sitio 1 (Quebrada Ganado)}

Este sitio se ubica sobre un flanco sur del cerro denominado Chiqueros en Quebrada Ganado de Tárcoles, en el cantón de Garabito de Puntarenas, localizándose, específicamente, en la divisoria de aguas de la microcuenca de la quebrada Chiqueros y la microcuenca de la quebrada

Cuadro 1

Numeración de los sitios que contempla el estudio

\begin{tabular}{ccc}
\hline Sitio & Nombre & Lugar \\
\hline 1 & Cerro Chiqueros & Quebrada Ganado \\
2 & El Castillo & Lago Arenal \\
3 & El Coco & Playas del Coco \\
4 & Santa Teresita & Zarcero \\
5 & La Sabana & San José \\
6 & Sánchez & Lomas de Ayarco \\
$7 \mathrm{~A}$ & Boulevart & Jacó \\
$7 \mathrm{~B}$ & Plaza Calle & Jacó \\
& Ancha & Pozos de Santa \\
8 & Naciente & Ana \\
\hline
\end{tabular}

Cañablancal. La pendiente en ambos flancos del lomo es fuerte generando un relieve pronunciado y con cambios abruptos desde el punto de vista topográfico. El uso de suelo se trata de pastos con parches irregulares de bosque en regeneración y secundario. El sitio específico donde se ha realizado el sondeo presenta una condición plana producto de antiguos movimientos de tierra antropogénicos

La geología de la zona se encuentra dominada por rocas sedimentarias de la Formación Punta Carballo. Esta formación está constituida por rocas siliciclásticas de tipo areniscas finas, medias y gruesas, lutitas, conglomerados y brechas (Linkimer \& Aguilar, 2000). Para el sitio específico de estudio se identifican una secuencia constituida por lutitas y areniscas, ligeramente carbonatadas, verdosas hasta café claro (Herrera, 1985; Leandro, 1985).

El análisis geológico local define la presencia de un macizo rocoso constituido por una secuencia de arenisca fina - lutita gris verdosa hasta café claro amarillento, con meteorización esferoidal tipo capa de cebolla en diferentes grados.

\section{Sitio 2 (Lago Arenal)}

Este sitio se ubica en la orilla del Lago Arenal, en los alrededores de El Castillo, en el distrito Peñas Blancas del cantón San Ramón de Alajuela. El sitio posee una pendiente moderada con un relieve ondulado, el cual se trata de un segmento lobular extendido del cerro conocido como Fila Cairo, localizado en un área al sur del lago Arenal. El uso del suelo en este sector se caracteriza por ser de tipo pastos con cobertura boscosa en las zonas riberinas de los ríos y quebradas, debido a las actividades tradicionales de la ganadería.

La geología de la zona está constituida por una secuencia de tefras en capas no consolidadas de depósitos plinianos y sub-plinianos asociados con erupciones del volcán Arenal, principalmente las capas AR-22 a la capa AR-3 (Soto \& Alvarado, 2006), con ausencia de algunas intermedias. Además, se presentan lavas sumamente 


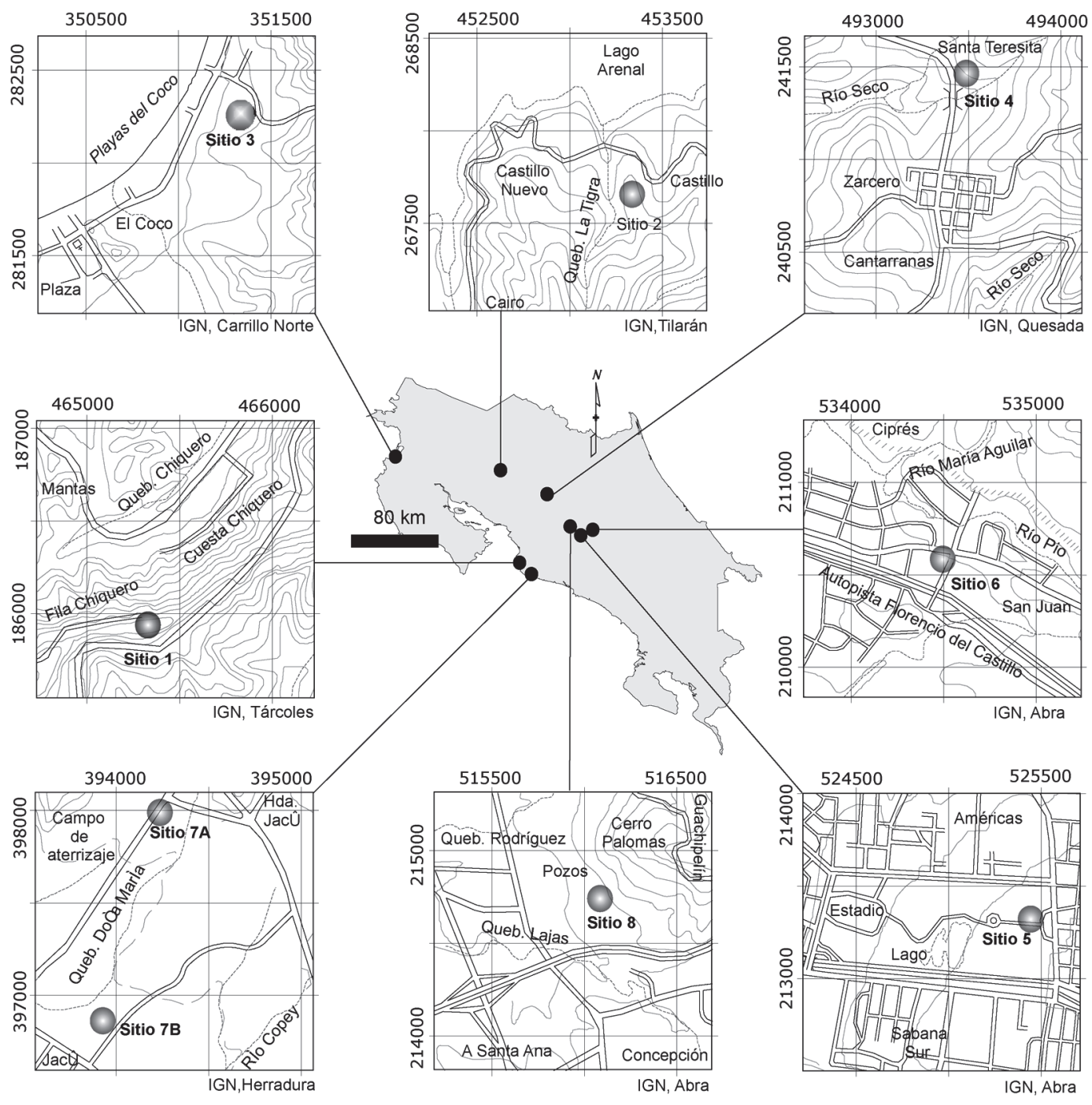

Fig. 2: Ubicación geográfica de los ocho sitios estudiados.

alteradas originadas en eventos eruptivos antíguos correlacionables con litologías de la Formación Monteverde, Grupo Aguacate.

El análisis geológico local específica la presencia de materiales volcánicos recientes con litologías asociadas a los materiales depositados por el volcán Arenal, en la parte superior, y materiales volcánicos terciarios de los cerros de Tilarán, en la parte inferior de la secuencia.

\section{Sitio 3 (Playas del Coco)}

Este sondeo se efectuó en Playas del Coco, aproximadamente $2 \mathrm{~km}$ noreste y 450 metros sureste de la iglesia católica de Playas del Coco, en el distrito de sardinal del cantón de Carrillo, Guanacaste. El sitio se localiza en la zona de baja pendiente de la planicie costera de la cuenca de Playas del Coco, en el sector de transición hacia 
el piedemonte y los cerros de Loma Jabillo. El uso del suelo es típicamente urbano en este sector, con tacotales hacia el sector montañoso.

De acuerdo con Denyer \& Arias (1993) en el sector de Playas de Coco afloran litologías asociadas con el Complejo de Nicoya y sedimentos aluviales costeros. El Complejo de Nicoya son rocas básicas con textura afanítica hasta ligeramente porfirítica de color verde oscuro a gris con fenocristales de plagioclasa, augitas y opacos; en algunos sectores se encuentran almohadillados y en otros sectores sencillos con textura "spinifex"

La alteración predominante es cloritización y óxidos de hierro, pero también aparecen vetillas de calcedonia, zeolitas, ópalo y algunas veces calcita. Tectónicamente se presentan muy fracturados y meteorizados. La edad máxima de esta litología es Jurásico.

De acuerdo con Gómez (2005) los sedimentos incluyen los depósitos de playa de origen fluvial y coluvial. Estos materiales han sido depositados en las partes bajas de la zona generando un relieve muy plano, por el arrastre y depositación de los materiales erosionados de los cerros adyacentes.

Vargas (2002) a partir de la información de pozos perforados describe una secuencia de interestratificaciones de capas arcillosas hasta gravosas de espesores variables entre 2-5 metros con una diferenciación marcada de materiales sumamente arcillosos después de los 15 metros.

Las tomografías eléctricas realizadas por Gómez (2005) demuestran la presencia de capas lenticulares de arcilla entre 9 y 25 metros.

\section{Sitio 4 (Zarcero)}

Este sitio se localiza en Santa Teresita en los alrededores de Zarcero, en el distrito del mismo nombre del cantón Alfaro Ruiz, Alajuela. La pendiente es moderada a alta con relieve pronunciado hacia el oeste dentro de la micro-cuenca del río Zarcero. El uso de suelo del sitio es de pasto en zona de cultivo con transición a área urbana.
Se identifican depósitos de material volcánico producto de erupciones plinianas, lahares, flujos piroclasticos y lavas asociados con la actividad de los cerros Palmira y Platanar.

Localmente se determina la presencia de tobas en la parte superior de la secuencia con ignimbritas poco soldadas en la parte inferior.

\section{Sitio 5 (San José)}

Este sitio se trata del parque metropolitano $\mathrm{La}$ Sabana. El ensayo se ha efectuado en las proximidades del edificio de la Federación Costarricense de Fútbol. El sitio se localiza en el distrito Mata Redonda, en el cantón central de San Jose. La pendiente es plana con cobertura de tipo pastos con arboles aislados.

La geología de la zona, de acuerdo con Denyer \& Arias (1991), está constituida por cenizas inconsolidadas, lahares e ignimbrita. Esta última, perteneciente a la formación Tiribí, típicamente constituidas por ignimbritas grises (Echandi, 1981).

Los lahares, también conocidos como la lavina del Valle Central, poseen su origen en el volcán Cabeza de Vaca, localizado en el macizo volcánico del Irazú (Hidalgo et al., 2004), y se encuentran constituidos por bloques de lava redondeados a sub-angulares flotantes en una matriz de lodos volcánicos arcillosos.

Por último, las cenizas inconsolidadas son producto de los últimos eventos de erupciones volcánicas registradas en el Valle Central (Denyer \& Arias, 1991).

\section{Sitio 6 (Lomas de Ayarco)}

Este sitio se trata de un sitio en las cercanías del Hipermas en Lomas de Ayarco Norte. Administrativamente, el sitio se localiza en el distrito Sánchez, cantón Curridabat, San José. La pendiente es baja formando un relieve desde plano hasta ondulado. La cobertura del suelo es urbana con parches de pastos con arboles aislados en lotes baldíos. 
La geología de la zona, está conformada por lahares y cenizas (Denyer \& Arias, 1991), ya descritos para el sitio anterior.

\section{Sitio 7 (Jacó)}

En este sitio se realizaron dos ensayos, el primero denominado 7a, se localiza en un sector de la carretera de acceso a Playa Jacó, precisamente por donde se ubica el Boulevart. El segundo sector, denominado, $7 \mathrm{~b}$ se ubica en una plaza de la calle ancha de acceso a Playa Jacó. Geográficamente, ambos sitios se localizan en el distrito Jacó, cantón Garabito, Puntarenas.

De acuerdo con la morfología de la cuenca ambos sectores se localizan en la planicie aluvial, que presenta un relieve plano. La cobertura del suelo en este sector es urbana con pastos y parches de tacotales.

La geología de esta cuenca, es especialmente, aluvial rodeada de sector montañoso conformado por rocas asociadas al Complejo de Nicoya y la Formación Punta Carballo.

El Complejo de Nicoya, constituido por basaltos toleiticos almohadillados, masivos, y en muchas ocasiones brechificados. Generalmente, tienen una textura afanitica y están compuestos por pequeños cristales aciculares de plagioclasa, granos de augita y pequeñas cantidades de magnetita y a veces de olivino alterado (Kussmaul, 2000).

Por otro lado, la Formación Punta Carballo, está constituida principalmente por areniscas finas, muy fosilíferas, con escasas lodolitas, lutitas, brechas y conglomerados de ambiente litoral (Laurito, 1988).

Los sedimentos aluviales en este sector están constituidos por capas de origen marino tipo litoral y depósitos terrígenos coluvio-aluviales (Arias, 1996).

\section{Sitio 8 (Pozos de Santa Ana)}

Este sitio se localiza en el lugar conocido como Naciente, en las cercanías de Pozos del cantón Santa Ana, San José. Las pendientes de la zona son moderadas a bajas, donde el sitio se localiza en el sector transicional de la zona plana al piedemonte.

Geológicamente, este sitio se ubica dentro de lo mapeado como Formación Pacacua (Denyer \& Arias, 1991). Se trata de una secuencia volcaniclástica de granulometría diversa dispuestas como estratificaciones decimétricas hasta métricas de areniscas vulcaniclasticas finas, gruesas y guijarrosas; además se asocian con esta formación tobas, tobitas y brechas finas y gruesas. Los colores predominantes de estas rocas son rojos y morados, aunque el verde es llamativo apareciendo aisladamente. Además, en zonas cercanas afloran las rocas de la Formación Grifo Alto constituidas por una serie de lavas andesiticas y flujos piroclásticos de la misma composición; en algunos casos aislados se observan basaltos y dacitas. Por otro lado, la litología que recubre la zona plana se trata de la Formación Tiribí, descrita en el sitio 5.

\section{CORRELACION GEOLOGICA DE LA VELOCIDAD DE ONDA CORTANTE}

Los resultados de los sondeos SASW realizados en los ocho sitios demuestran fuertes contrastes en la velocidad de onda cortante para los diferentes materiales. Se han descrito las columnas estratigráficas asociadas para cada sitio, de acuerdo a las observaciones de campo, y en algunos casos, se ha debido recurrir a la búsqueda de información litológica en la base de pozos del SENARA. Los sitios constituidos, principalmente, por macizos rocosos, presentan descripciones directas de parámetros como RMR (Bieniawski, 1989), GSI (Hoek \& Brown, 1997) con su modificación (Marinos \& Hoek, 2000) y resistencia a la compresión simple con ensayos puntuales (ISRM, 1981). En el caso, de los sedimentos no consolidados, tanto aluviales como volcánicos recientes, se presentan descripciones de valores de Nspt promedio (para las capas clasificadas como suelos), de un sondeo comprobatorio realizado en el sitio. 


\section{Sitio 1 (Quebrada Ganado)}

En Cerro Chiqueros se destaca la presencia de 7 metros de roca fuertemente meteorizada con fluctuaciones internas de la velocidad de onda de $150 \mathrm{~m} / \mathrm{s}$ hasta más de $1000 \mathrm{~m} / \mathrm{s}$, provocadas por la presencia de bloques métricos de meteorización esferoidal tipo capas de cebolla. Posteriormente, de 7 a 14 metros de profundidad la velocidad de onda disminuye y permanece más constante desde $200 \mathrm{~m} / \mathrm{s}$ hasta poco más de $500 \mathrm{~m} / \mathrm{s}$. Este sector está constituido por roca moderadamente meteorizada. Luego, de 14 a 20 metros la velocidad de onda aumenta manteniendo cerca de $750 \mathrm{~m} / \mathrm{s}$ debido al cambio litológico hacia la roca levemente meteorizada. Al final de la secuencia aflora la roca fractura, sin embargo la velocidad de onda no presenta mayores variaciones con una velocidad alrededor de $700 \mathrm{~m} / \mathrm{s}$. Es claro que las variaciones de la velocidad de onda están fuertemente relacionadas con el efecto de la meteorización esferoidal tipo capas de cebolla, denotando disminuciones abruptas en el perfil de velocidad producto de la presencia de matriz de roca meteorizada alrededor de los bloques redondeados de roca más compacta (Fig. 3). Los valores del RMR manifiestan una clasificación como macizo rocoso de muy mala calidad en los primeros 21 metros, variando a un macizo rocoso de mala calidad en las capas siguientes (Cuadro 2). El valor de Vs30 definida para este sitio es de $830 \mathrm{~m} / \mathrm{s}$ con un periodo natural de 0,22 .

\section{Sitio 2 (Lago Arenal)}

Para el sector de El Castillo se presentan rasgos relevantes, principalmente por la variación drástica de la velocidad de onda en los primeros ocho metros (Fig. 4), debido a la presencia de capas intercaladas de ceniza y tobas más o menos compactadas (Cuadro 3). Estas variaciones también se observan en el ensayo SPT efectuado en el sitio, el cual promedia un Nspt de 10. En los primeros $2 \mathrm{~m}$, la velocidad de onda cortante varía entre 100 hasta $300 \mathrm{~m} / \mathrm{s}$ asociado con una

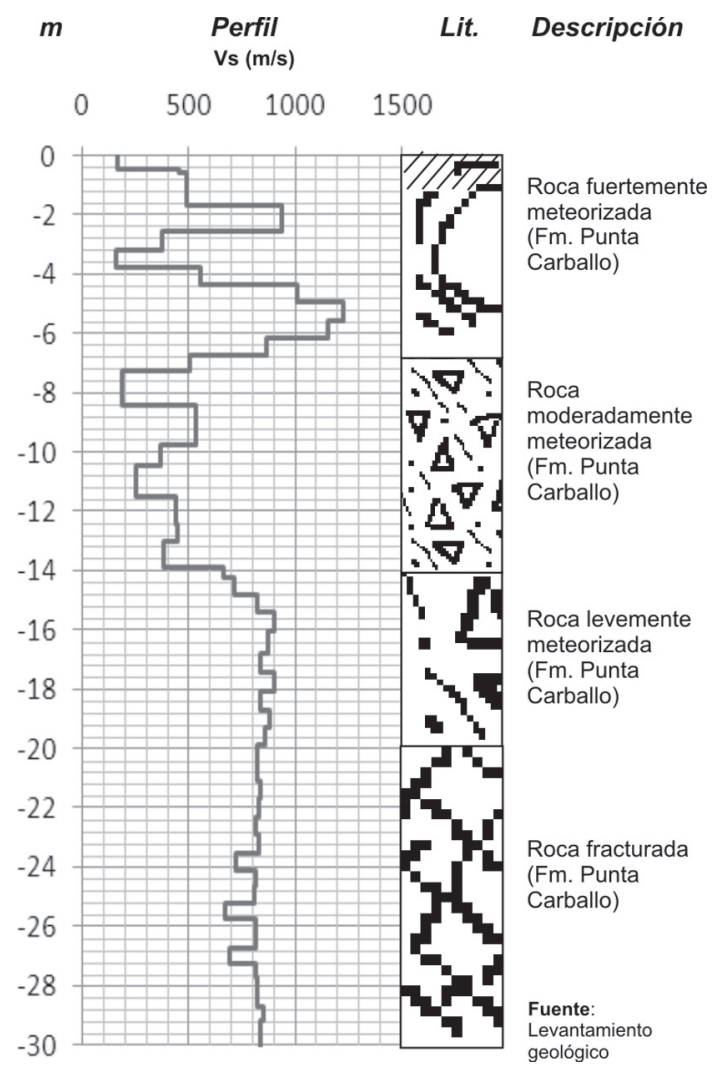

Fig. 3: Columna geológica correlativa con Vs para el sitio 1

capa de ceniza. Entre 2 y 8 metros de profundidad se observan fluctuaciones entre 400 y 250 $\mathrm{m} / \mathrm{s}$, debido a la intercalación de tobas y cenizas. La aparición de una toba de lapilli genera una variación en la velocidad de onda cortante,

Cuadro 2

Correlación de valores promedios de Vs y parámetros de la roca para sitio 1

\begin{tabular}{ccccc}
\hline Prof $(\mathrm{m})$ & Vs $(\mathrm{m} / \mathrm{s})$ & RMR & GSI & R \\
\hline $0-1$ & 200 & 1 & $10-15$ & R0 \\
$1-6$ & 900 & 7 & $20-25$ & R1 \\
$6-13$ & 400 & 16 & $25-30$ & R1 \\
$13-21$ & 800 & 20 & $30-35$ & R2 \\
$21-?$ & 800 & 31 & $40-45$ & R3 \\
\hline
\end{tabular}


la cual se mantiene alrededor de $450 \mathrm{~m} / \mathrm{s}$, entre 8 y 21 bajo el nivel del suelo. Al final, se observa la aparición de una lava sumamente alterada que genera una disminución de la Vs hasta cerca de $400 \mathrm{~m} / \mathrm{s}$. La factor Vs30 calculado para este sitio es de $419 \mathrm{~m} / \mathrm{s}$ para un periodo natural de 0,33 .

\section{Sitio 3 (Playas del Coco)}

Para este sitio en Playas del Coco, los primeros tres metros constituidos por capas de arenas, provocan la presencia de velocidades bajas (Cuadro 4), entre 200 y $450 \mathrm{~m} / \mathrm{s}$. Una capa de grava de origen coluvial aumenta la velocidad a más de $1000 \mathrm{~m} / \mathrm{s}$ de 3 a $9 \mathrm{~m}$ de profundidad (Fig. 5). La secuencia continua, de 9 a $14 \mathrm{~m}$, con una capa de arcilla con velocidad baja $(150 \mathrm{~m} / \mathrm{s})$, muy constante. A partir de los $16 \mathrm{~m}$ aparecen los basaltos del Complejo de Nicoya con valores de Vs cercanos a $1000 \mathrm{~m} / \mathrm{s}$ con algunos sectores de mayor fracturación donde disminuye la velocidad. Entre 14 y 16 metros los basaltos forman un paleo-suelo con una Vs entre 600 y $700 \mathrm{~m} / \mathrm{s}$. El parámetro Vs30 obtenido es de $491 \mathrm{~m} / \mathrm{s}$ con un periodo natural de 0,25 .

\section{Sitio 4 (Zarcero)}

En este caso, el sondeo realizado en Santa Teresita, define la presencia de $2 \mathrm{~m}$ de suelo que mantiene una velocidad de onda alrededor de los 150 m/s (Fig. 6). Posteriormente, la toba cenicienta y la ignimbrita se comportan muy homogéneamente con velocidades cercanas a los $250 \mathrm{~m} / \mathrm{s}$, esto probablemente por la fuerte alteración de la ignimbrita, que crea un comportamiento dinámico similar a una toba (Cuadro 5). El factor Vs30 está dado en $280 \mathrm{~m} / \mathrm{s}$ con un periodo natural de 0,43.

\section{Sitio 5 (San José)}

De acuerdo con este sondeo, en el sector de La Sabana (Fig. 7), se interpreta la presencia de un suelo blando de un metro de espesor con una velocidad menor a $100 \mathrm{~m} / \mathrm{s}$, seguido por una toba compacta de 1 a $5 \mathrm{~m}$ de profundidad con una velocidad cercana a los $400 \mathrm{~m} / \mathrm{s}$ (Cuadro 6). Entre 7 y $14 \mathrm{~m}$ de profundidad se presenta un lahar con una velocidad cercana a $\operatorname{los} 1000 \mathrm{~m} / \mathrm{s}$, con disminución de la velocidad hacia el contacto superior e inferior de la capa, cercano a los $600 \mathrm{~m} / \mathrm{s}$, posiblemente siendo un indicador de una acumulación de bloques hacia la parte central del lahar. Después de los $14 \mathrm{~m}$ aparece la ignimbrita de la Formación Tiribícon una velocidad típica de $600 \mathrm{~m} / \mathrm{s}$, muy continua y homogénea en el comportamiento dinámico. El valor Vs30 alcanzado es de 502, con un periodo natural calculado de 0,26.

\section{Sitio 6 (Lomas de Ayarco)}

En este perfil de velocidad de onda, presenta muchas fluctuaciones en los primeros $24 \mathrm{~m}$ asociándose con la presencia de intercalaciones consolidadas y no consolidadas de capas epiclasticas (Cuadro 7). Estas intercalaciones provocan variaciones en la velocidad de onda entre $275 \mathrm{~m} / \mathrm{s}$ hasta $600 \mathrm{~m} / \mathrm{s}$ en capas de 2 hasta $12 \mathrm{~m}$ de espesor (Fig. 8). En los primeros $2 \mathrm{~m}$ se observa un suelo con una velocidad de $200 \mathrm{~m} / \mathrm{s}$ y posteriormente la presencia de una toba aumenta la velocidad a 400 $\mathrm{m} / \mathrm{s}$ hasta los 5 metros. Desde 5 hasta 17 metros la presencia de una arcilla con bloques (posible lahar soportando en la matriz arcillosa) genera fluctuaciones en la Vs entre 350 y $550 \mathrm{~m} / \mathrm{s}$. La aparición de una ceniza, de 17 a $20 \mathrm{mbns}$, disminuye la velocidad a $300 \mathrm{~m} / \mathrm{s}$ y la existencia de una 
Cuadro 3

Correlación de valores promedios de Vs y parámetros de la roca para sitio 2

\begin{tabular}{cccc}
\hline Prof $(\mathrm{m})$ & Vs $(\mathrm{m} / \mathrm{s})$ & Nspt & $\mathrm{R}$ \\
\hline $0-1$ & 100 & 4 & $\mathrm{~S} 2$ \\
$2-9$ & 300 & 10 & $\mathrm{~S} 4$ \\
$9-22$ & 420 & & $\mathrm{~S} 6$ \\
$22-?$ & 400 & & $\mathrm{R} 1$ \\
\hline
\end{tabular}

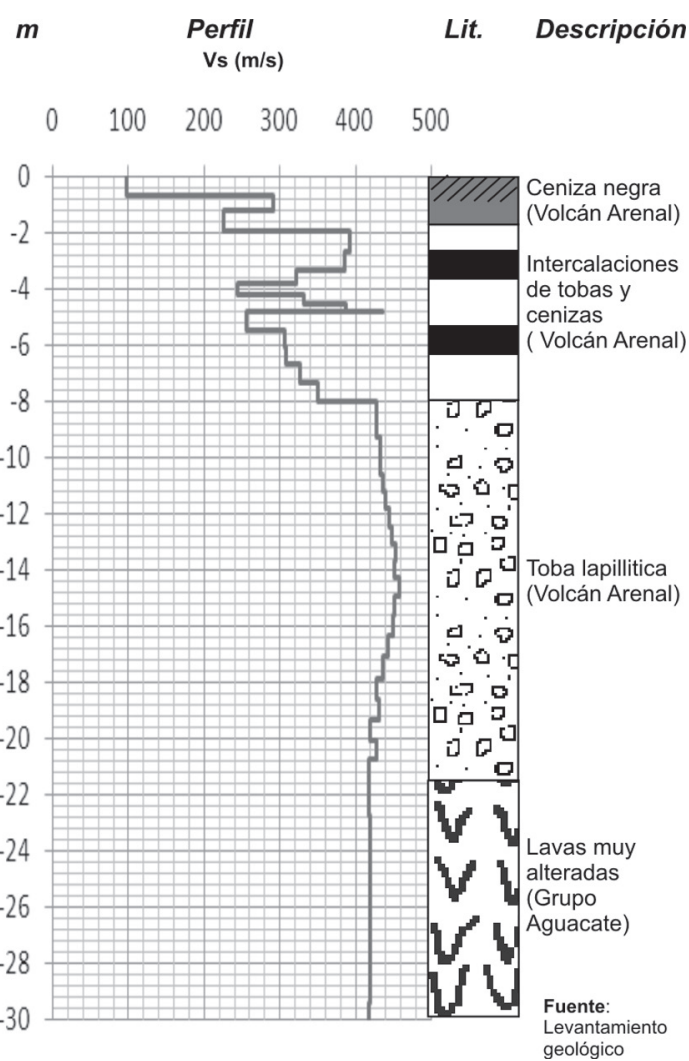

Fig. 4: Columna geológica correlativa con Vs para el sitio 2.
Cuadro 4

Correlación de valores promedios de Vs y parámetros del sustrato para sitio 3

\begin{tabular}{ccc}
\hline Prof $(\mathrm{m})$ & Vs $(\mathrm{m} / \mathrm{s})$ & Nspt \\
\hline $0-3$ & 250 & 8 \\
$3-9$ & 1000 & \\
$9-14$ & 140 & \\
$14-16$ & 600 & \\
$16-?$ & 1100 & \\
\hline
\end{tabular}

m

Perfil

Lit. Descripción

Vs $(\mathrm{m} / \mathrm{s})$

$0 \quad 500 \quad 1000 \quad 1500$

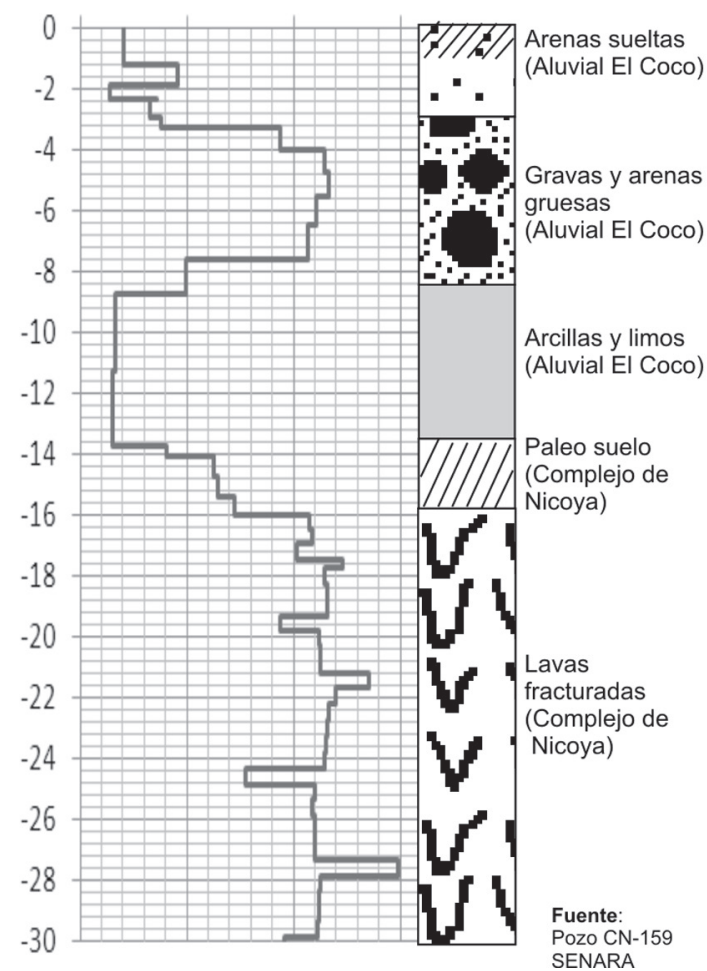

Fig. 5: Columna geológica correlativa con Vs para el sitio 3. 
arcilla compacta de 20 a 24, provoca una velocidad de onda de $200 \mathrm{~m} / \mathrm{s}$. A partir de los 24 metros de profundidad, la velocidad aumenta a $800 \mathrm{~m} / \mathrm{s}$. Este cambio de velocidad se asocia con el cambio litológico del lahar. El valor de la Vs30 es de 471 $\mathrm{m} / \mathrm{s}$ con un periodo natural de 0,35 .

\section{Sitio 7 (Jacó)}

En este sitio se realizaron dos sondeos. Ambos perfiles demuestran la presencia de capas aluviales no consolidadas. En el sondeo 7A (Cuadro 8), se interpreta la existencia de materiales más homogéneos con pocas variaciones (Fig. 9), encontrando arenas, limos y arenas compactas con leves cambios en la velocidad de onda, entre 160 y $200 \mathrm{~m} / \mathrm{s}$. Mientras que el sitio 7B si presenta una composición más heterogénea, principalmente por la presencia de una capa con velocidad de onda muy baja de 2-6 m de profundidad (Fig. 10), relacionada con una arcilla gris suave (Cuadro 9). En este último caso, la velocidad de onda se encuentra cercana a los $200 \mathrm{~m} / \mathrm{s}$ en los primeros $2 \mathrm{~m}$ de suelo, luego del cual disminuye a 70 y $50 \mathrm{~m} / \mathrm{s}$ por la presencia de una arcilla blanda entre 2 y $6 \mathrm{~m}$ de profundidad. La arcilla se vuelve más compacta de 6 a $8 \mathrm{~m}$ con una velocidad de onda cortante cercana a los $125 \mathrm{~m} / \mathrm{s}$. La presencia de una arena densa entre 8 y $12 \mathrm{~m}$ aumenta la Vs entre 240 y $180 \mathrm{~m} / \mathrm{s}$. Entre 12 y $30 \mathrm{~m}$ la velocidad se mantiene muy constante en $160 \mathrm{~m} / \mathrm{s}$ asociada a la existencia de una capa de arena. El valor de Vs30 obtenido para el sitio 7A es de $246 \mathrm{~m} / \mathrm{s}$, disminuyendo para el sitio $7 \mathrm{~B}$ a $214 \mathrm{~m} / \mathrm{s}$. Los periodos naturales obtenidos son de 0,50 y 0,61 , respectivamente.

\section{Sitio 8 (Pozos de Santa Ana)}

En este sitio, el perfil indica la presencia de una capa de suelo poco compacta de un metro de espesor (Cuadro 10), con una Vs cercana a 100 $\mathrm{m} / \mathrm{s}$, después de la cual en una posición inferior de 1 hasta 4 metros se identifica un conglomerado arenoso meteorizado con una velocidad de $500 \mathrm{~m} / \mathrm{s}$. Desde 4 hasta 8 se asocia la velocidad cercana a $200 \mathrm{~m} / \mathrm{s}$ con un estrato de lutita café oscura muy friable. Luego, una velocidad cercana a $600 \mathrm{~m} / \mathrm{s}$ se debe a una arenisca media meteorizada. Posterior a este estrato, entre 11 y 30 metros, la velocidad de $1000 \mathrm{~m} / \mathrm{s}$ se asocia con un conglomerado en estado sano (Fig. 11). El valor de Vs30 en este sitio es de $473 \mathrm{~m} / \mathrm{s}$ con un periodo natural de 0,27 .

\section{APLICACIÓN DEL SASW A LA INGENIERÍA DE CIMENTACIONES}

Las condiciones geológicas y geotécnicas particulares de todos los sitios muestran variaciones importantes en los valores de los parámetros dinámicos necesarios para la ingeniería estructural.

El comentario al Código Sísmico de Costa Rica (2002), establece cuatro diferentes tipos de sitios de cimentación, clasificados de acuerdo a la velocidad de onda cortante en los primeros 30 metros. Esta característica, conocida como Vs30, cuantifica un valor promedio calculado de acuerdo con la formula:

$$
V_{s(30)}=\frac{30}{\sum(h i / V s i)}
$$

Donde hi y vi son las velocidades respectivas para cada estrato que compone los primeros $30 \mathrm{~m}$.

La clasificación del Vs30 (Cuadro 11) para los ocho sitios demuestra valores en un rango superior a $214 \mathrm{~m} / \mathrm{s}$ e inferior a $830 \mathrm{~m} / \mathrm{s}$ para sitios caracterizados como $\mathrm{S} 2$, típicamente, aunque un sitio se clasifica como S1 y dos como S3.

Otra variable importante a la hora de la definición de los parámetros dinámicos del suelo es el valor conocido como periodo natural del suelo, que se define mediante la ecuación: 
Cuadro 5

Correlación de valores promedios de Vs y parámetros del sustrato para sitio 4

\begin{tabular}{cccc}
\hline Prof $(\mathrm{m})$ & Vs $(\mathrm{m} / \mathrm{s})$ & Nspt & $\mathrm{R}$ \\
\hline $0-2$ & 160 & 2 & $\mathrm{~S} 2$ \\
$2-11$ & 250 & 8 & S5 \\
$22-?$ & 250 & & R0 \\
\hline
\end{tabular}

m

Perfil

Vs $(\mathrm{m} / \mathrm{s})$

Lit. Descripción

$\begin{array}{lllll}0 & 100 & 200 & 300 & 400\end{array}$

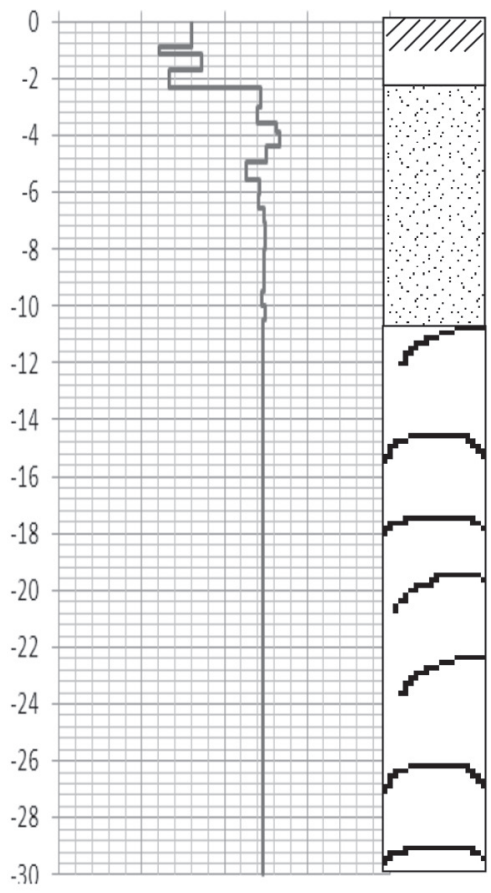

Suelo

Toba

(Volcánico reciente

Cerro Palmira-

Porvenir)

Ignimbrita

(Volcánico reciente

Cerro Palmira-

Porvenir)

Fig. 6: Columna geológica correlativa con Vs para el sitio 4.
Cuadro 6

Correlación de valores promedios de Vs y parámetros del sustrato para sitio 5

\begin{tabular}{ccc}
\hline Prof $(\mathrm{m})$ & Vs $(\mathrm{m} / \mathrm{s})$ & Nspt \\
\hline $0-1$ & 100 & 5 \\
$1-5$ & 350 & 20 \\
$5-7$ & 600 & 40 \\
$7-14$ & 850 & \\
$14-?$ & 600 & \\
\hline
\end{tabular}

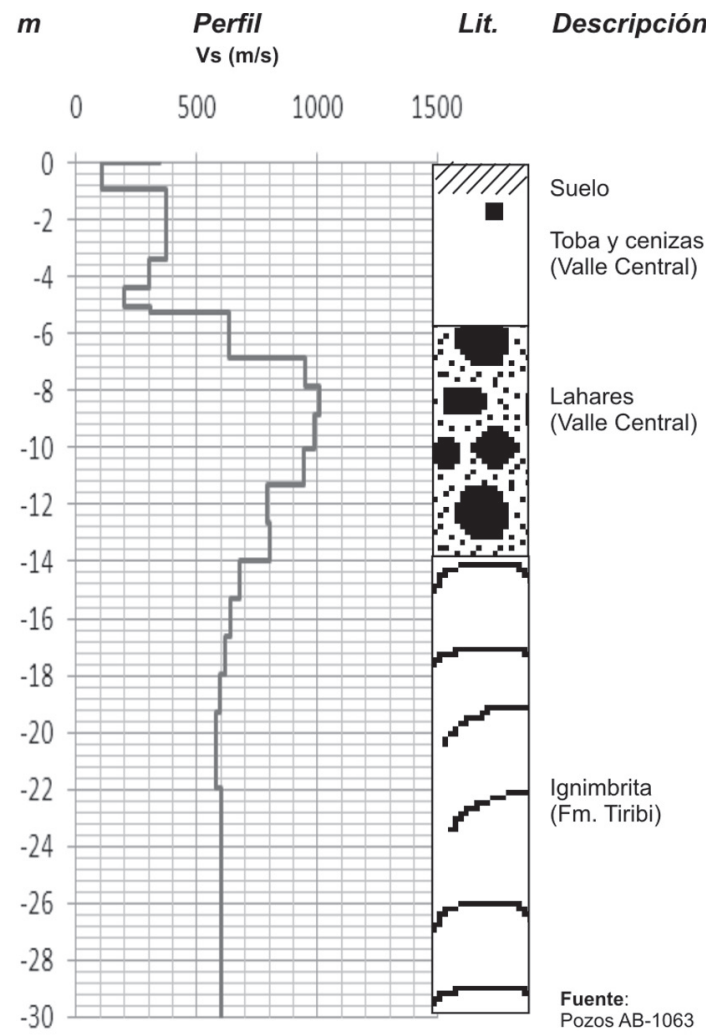

Fig. 7: Columna geológica correlativa con Vs para el sitio 5. 
Cuadro 7

Correlación de valores promedios de Vs y parámetros del sustrato para sitio 6

\begin{tabular}{ccc}
\hline Prof $(\mathrm{m})$ & Vs $(\mathrm{m} / \mathrm{s})$ & Nspt \\
\hline $0-2$ & 180 & 2 \\
$2-5$ & 275 & 12 \\
$5-17$ & 400 & 20 \\
$17-20$ & 375 & \\
$20-24$ & 275 & \\
$14-40$ & 800 & \\
\hline
\end{tabular}

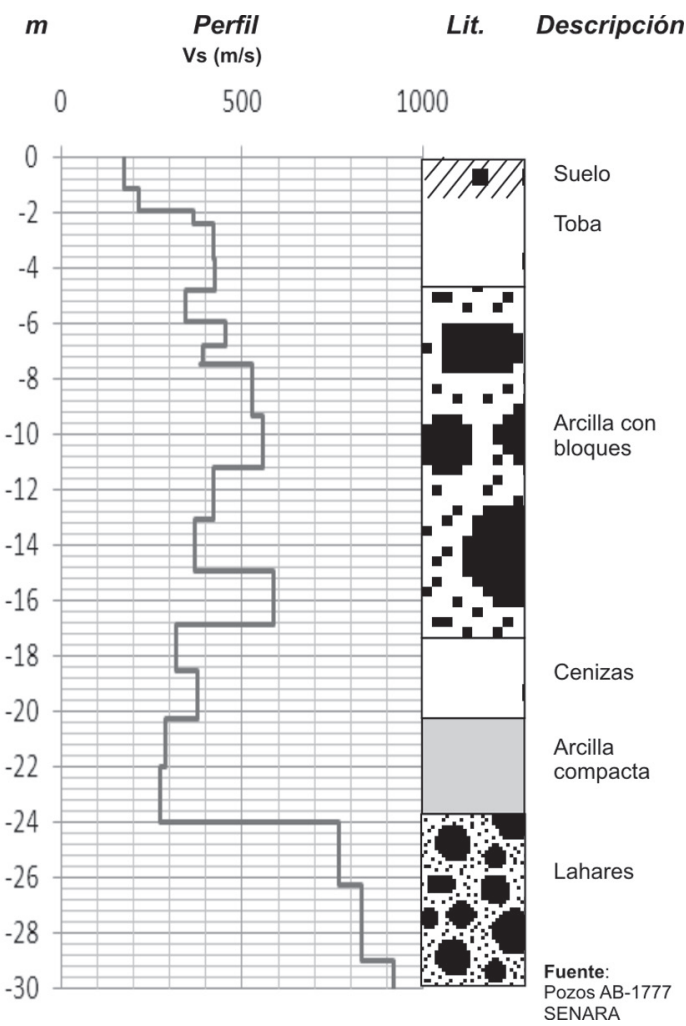

Fig. 8: Columna geológica correlativa con Vs para el sitio 6.
Cuadro 8

Correlación de valores promedios de Vs y parámetros del sustrato para sitio $7 \mathrm{~A}$

\begin{tabular}{ccc}
\hline Prof $(\mathrm{m})$ & Vs $(\mathrm{m} / \mathrm{s})$ & Nspt \\
\hline $0-1$ & 200 & 6 \\
$1-2.5$ & 100 & 2 \\
$2.5-9$ & 170 & 20 \\
$9-11$ & 150 & \\
$11-30$ & 180 & \\
\hline
\end{tabular}

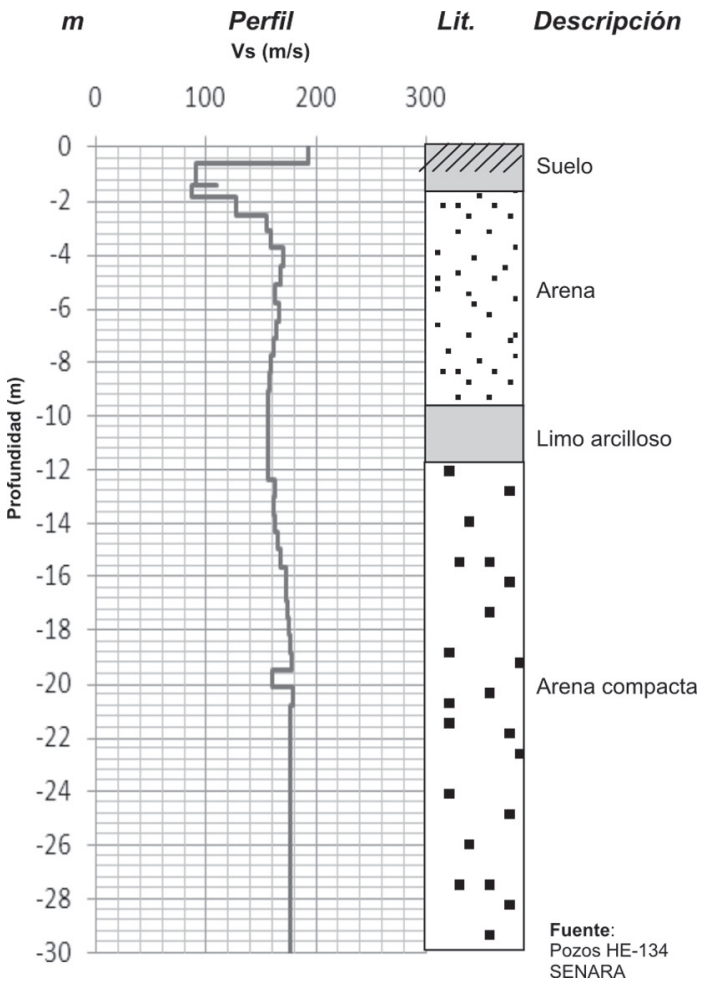

Fig. 9: Columna geológica correlativa con Vs para el sitio 7a. 
Cuadro 9

Correlación de valores promedios de Vs y parámetros del sustrato para sitio $7 \mathrm{~B}$

\begin{tabular}{ccc}
\hline Prof $(\mathrm{m})$ & Vs $(\mathrm{m} / \mathrm{s})$ & Nspt \\
\hline $0-1.5$ & 180 & 6 \\
$1.5-6$ & 60 & 1 \\
$6-8$ & 120 & 4 \\
$8-13$ & 300 & 20 \\
$13-30$ & 260 & \\
\hline
\end{tabular}

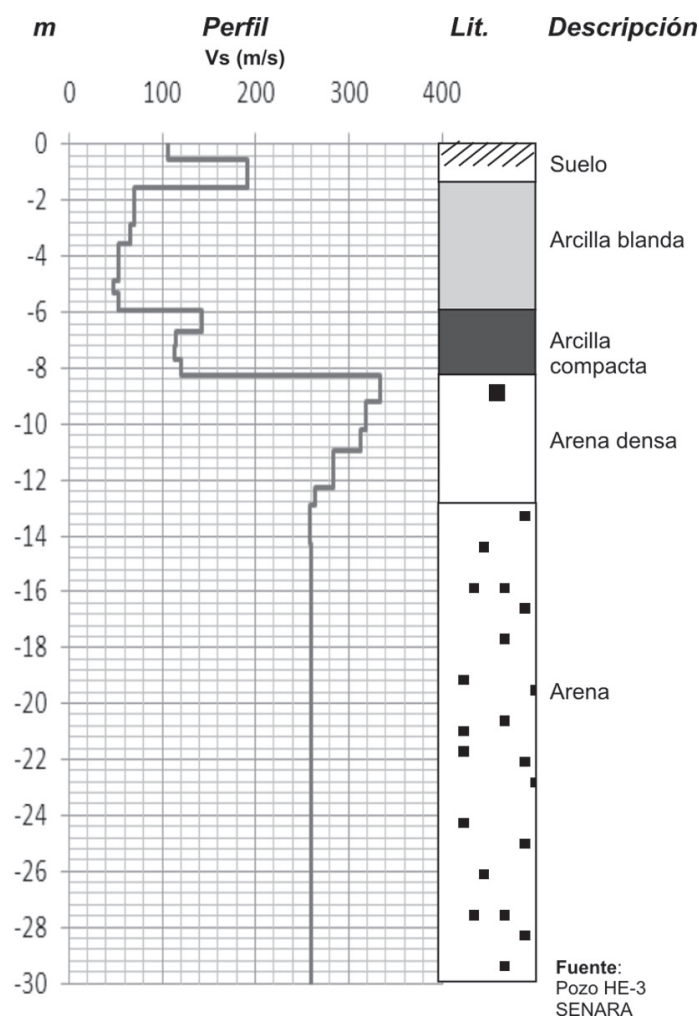

Fig. 10: Columna geológica correlativa con Vs para el sitio $7 \mathrm{~b}$.
Cuadro 10

Correlación de valores promedios de Vs y parámetros de la roca para sitio 8

\begin{tabular}{cccccc}
\hline $\begin{array}{c}\text { Prof } \\
(\mathrm{m})\end{array}$ & $\begin{array}{c}\text { Vs } \\
(\mathrm{m} / \mathrm{s})\end{array}$ & Nspt & RMR & GSI & R \\
\hline $0-1$ & 100 & 6 & & & \\
$1-4$ & 500 & & 27 & $35-40$ & R2 \\
$4-8$ & 200 & & 12 & $25-30$ & R1 \\
$8-11$ & 550 & & 31 & $40-45$ & R3 \\
$11-30$ & 1000 & & 46 & $55-60$ & R4 \\
\hline
\end{tabular}

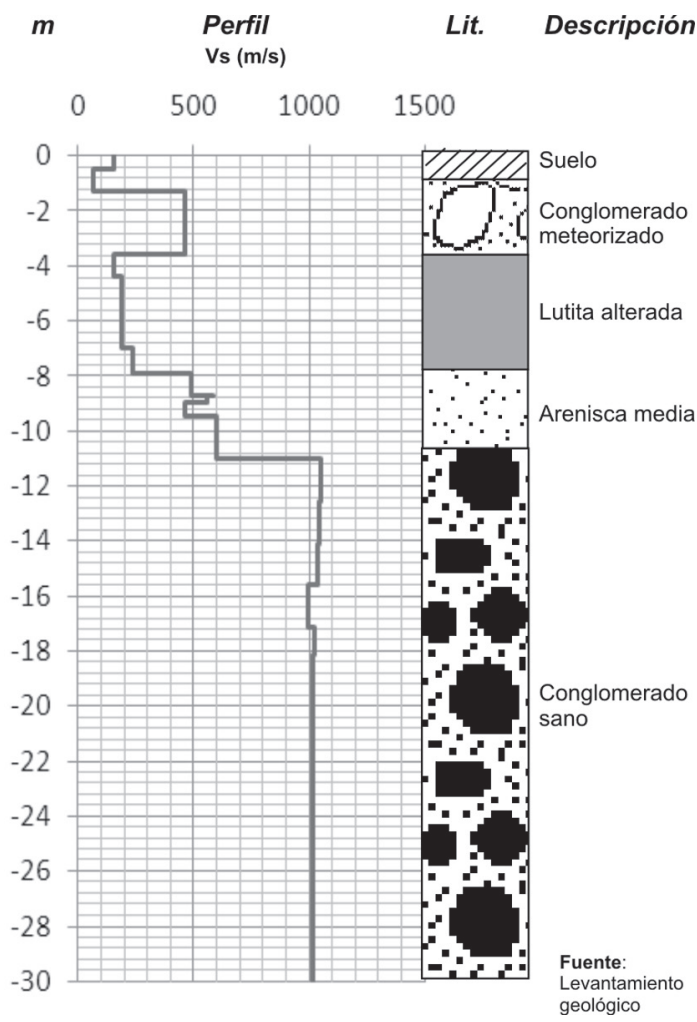

Fig. 11: Columna geológica correlativa con Vs para el sitio 8 . 


$$
T_{n}=4 \sum\left(h_{i} / V s_{i}\right) \quad(5
$$

Donde hi y vi son las velocidades respectivas para cada estrato que compone los primeros $30 \mathrm{~m}$.

El periodo natural del suelo posee un rango entre $0,22 \mathrm{~s}$ y $0,61 \mathrm{~s}$, siendo los sedimentos aluviales cuaternarios los que presentan periodos naturales más altos (Cuadro 11), mientras que los sitios constituidos por rocas sedimentarias son los que presentan periodos naturales más bajos.

Las variaciones marcadas tanto en el valor de la Vs30 como en el periodo natural se deben a las fuertes diferencias estratigráficas de todos los sitios, con presencia de capas blandas intercaladas con capas rígidas. Además, otro factor importante es la diferencia en la calidad del material, tanto en el grado de meteorización y alteración de la roca o el grado de compactación y soldamiento de sustratos no consolidados. Esto último, puede ser el argumento más claro para explicar la razón de las diferencias para estratos y capas con la misma composición geológica.

\section{CONCLUSIONES}

La técnica AEOS, análisis espectral de ondas superficiales (SASW), se convierte en un instrumento sumamente valioso para la determinación de las características dinámicas del suelo, generando perfiles de rigidez del material, con una correlación muy precisa asociada a la secuencia estratigráfica.
Los ocho sitios seleccionados presentan características geológicas muy particulares para cada uno, constituidos por materiales no consolidados (aluviales y tefras) y consolidados tanto volcánicos (lavas) como sedimentarios (macizos rocosos estratificados). Esta diversidad litológica permite la correlación directa de la velocidad de onda con la secuencia geológica, convirtiéndose el perfil de la velocidad de onda cortante en el "esqueleto" de la columna estratigráfica.

Del análisis correlativo, para los sitios estudiados, se obtiene que los sedimentos arcillosos $\mathrm{y}$ arenosos no consolidados poseen velocidades entre 50 y $200 \mathrm{~m} / \mathrm{s}$. Mientras que sedimentos más gruesos y consolidados como arenas compactas y gravas mantienen velocidades entre 250 y 800 $\mathrm{m} / \mathrm{s}$. Por otro lado, los lahares poseen velocidades en un rango típico de 800 a $1000 \mathrm{~m} / \mathrm{s}$. Las tobas cenicientas y tobas de lapilli entre 200 y $400 \mathrm{~m} / \mathrm{s}$. Las ignimbritas de la formación Tiribi, poseen una velocidad de onda típica cercana a $600 \mathrm{~m} / \mathrm{s}$, mientras que las lavas del Complejo de Nicoya, mantienen una velocidad cercana a los $1000 \mathrm{~m} / \mathrm{s}$. Las diferencias en la velocidad de onda entre estratos geológicos similares se deben a la calidad del material, tanto en el grado de meteorización y alteración de la roca o el grado de compactación y soldamiento de sustratos no consolidados.

La clasificación de los parámetros dinámicos del suelo para cada área indica la predominancia de sitios con clasificación S2, aunque dos sitios son de tipo S3 y solo uno posee clasi-

Cuadro 11

Características dinámicas de los sitios estudiados

\begin{tabular}{cccccc}
\hline SITIO & Lugar & Vs 30 & Tn & TIPO & GEOLOGIA \\
\hline 1 & Q. Ganado & 830 & 0,22 & S1 & Roca sedimentaria meteorizada terciaria \\
2 & Arenal & 419 & 0,33 & S2 & Tobas de lapilli recientes \\
3 & El Coco & 491 & 0,25 & S2 & Sedimentos aluviales y lavas cretácicas \\
4 & Zarcero & 280 & 0,43 & S3 & Tobas cenicientas cuaternarias \\
5 & La Sabana & 502 & 0,26 & S2 & Tobas y lahares cuaternarios \\
6 & Ayarco & 471 & 0,35 & S2 & Cenizas y lahares cuaternarios \\
7 A & Jacó & 246 & 0,50 & S3 & Sedimentos aluviales cuaternarios \\
$7 B$ & Jacó & 214 & 0,61 & S3 & Sedimentos aluviales cuaternarios \\
8 & Pozos & 473 & 0,27 & S2 & Rocas sedimentarias interestratificadas terciarias \\
\hline
\end{tabular}


ficación S1. El valor del parámetro Vs30 máximo encontrado corresponde con una secuencia sedimentaria de roca meteorizada con valor de $830 \mathrm{~m} / \mathrm{s}$, mientras que los valores más bajos se encuentran en sedimentos aluviales inconsolidados con 214 y $246 \mathrm{~m} / \mathrm{s}$.

\section{AGRADECIMIENTOS}

Se agradece a Wayner Fallas por toda su colaboración en la elaboración de los sondeos y los detalles experimentales para la aplicabilidad del método. Igualmente, se le agradece, al geólogo Gerardo J. Soto, todas las sugerencias brindadas respecto a esta investigación.

\section{REFERENCIAS}

ARIAS, M., 1996: Evaluación del riesgo a la contaminación del acuífero de Jacó, cantón de Garabito, Puntarenas.- 48 págs. SENARA. San José.

BIENIAWSKI, Z.T., 1989: Engineering rock mass clasifications.- 251 págs. Ed. Jonh Wiley and Sons, Inc. New York, USA.

\section{COLEGIO FEDERADO DE INGENIEROS} Y ARQUITECTOS, 2007: Comentarios sobre el Código Sísmico de Costa Rica, 2002.- 256 págs. Ed. Tecnológica de Costa Rica. Cartago.

CUÉllAR, V., MUÑOZ, F. \& VALERIO, J., 1995: Evaluación de la mejora del terreno en ingeniería civil mediante el análisis de ondas superficiales.- Física de la Tierra, 7: 259-280.

DENYER, P., \& ARIAS, O., 1991: Estratigrafía de la región Central de Costa Rica.- Rev. Geol. de Amér. Central, 12:1-59.

DENYER, P. \& ARIAS, O., 1993: Geología del norte de la Península de Nicoya, Costa Rica.Rev. Geol. de Amér. Central,16: 69-84.
ECHANDI, E., 1981: Unidades volcánicas de la vertiente norte de la cuenca del rio Virilla.123 págs. Univ. de Costa Rica, San José [Tesis Lic.].

GÓMEZ, M., 2005: Estudio hidrogeológico integral de las cuencas hidrográficas El Coco y Ocotal, Carrillo Guanacaste.- 110 págs. Univ. de Costa Rica, San José [Tesis M.Sc.].

HERRERA, D., 1985: Estudio geológico de la zona comprendida entre el pueblo Quebrada Ganado y Cerro Chiquero, provincia de Puntarenas.- 35 págs. Univ. de Costa Rica, San José [Inf. Campaña Geol.].

HIDALGO, P., ALVARADO, G.E. \& LINKIMER, L., 2004: La lavina del Valle Central (Costa Rica): lahar o debris avalanche?.-Revista Geológica de América Central, 30: 101109. San José

HOEK, E. \& BROWN, E.T., 1997: Practical estimates of rock mass strength.- Int. Journal of Rock Mechanics and Mining Sciences. 34(8):1165-1186.

INTERNATIONAL SOCIETY FOR ROCK MECHANICS (ISRM), 1981: Suggested methods for rock characterization, testing and monitoring.- 211 págs. Ed. E.T. Brown, Pergamon Press.Toronto, Canada.

KRAMER, S. L., 1996: Geotechnical Earthquake Engineering.- 653 págs. Ed. Prentice Hall. Inc. New Jersey, USA.

KUSSMAUL, S., 2000: Estratigrafía de las rocas igneas.- En: DENYER, P. \& KUSSMAUL, S. (eds): Geología de Costa Rica. Ed. Tecnológica de Costa Rica, Cartago, 133-154.

LAURITO, C., 1988: Análisis sedimentológico de las formaciones El Fraile, en la costa suroeste de Nicaragua, Carmen y Punta Carballo, en la costa pacífica de Costa Rica.- 117 págs. Univ. de Costa Rica, San José [Tesis Lic.]. 
LEANDRO, C., 1985: Geología del cerro Rincón Vellaco, Valle de Herradura y alrededores.31 págs. Univ. de Costa Rica, San José [Inf. Campaña Geol.].

LINKIMER, L. \& AGUILAR, T., 2000: Estratigrafía de las rocas sedimentarias.En: DENYER, P. \& KUSSMAUL, S. (eds): Geología de Costa Rica. Ed. Tecnológica de Costa Rica, Cartago, 101-132.

MARINOS, P. \& HOEK, E., 2000: GSI: A geologically friendly toll for rock mass strength estimations.- Proc. Geo Eng., I: 1422-1440.

MATTHEWS, M.C., HOPE, V.S. \& CLAYTON, C.R.I., 1996: The use of surface waves in the determination of ground stiffness profiles.- Proc. Inst. Civ. Engineers, Geotech. Engineering, 119: 84-95.

MOYA, A., CLIMENT, A., BOSCHINI, I., SCHMIDT, V., ROJAS, W., LINDHOLM, C., NADIM, F. \& ATAKAN, K., 2000: Microzonificación sísmica: temblores, fallas, crecimiento poblacional, periodos de suelos, espectros de respuesta.- 120 págs. Nonrad-Cepredenac. San José.

PÉREZ, J.M \& IZAGUIRRE, J, 1982: Desastres telúricos y tectónicos. -42 págs. Programa de agua potable y disposición de aguas residuales en situaciones de emergencia. CEPIS. OSP, Washingthon.

RAMÍREZ, R., SANTANA, G. \& CHACON, O., 1996: Mapa de amplificación sísmica del Valle Central, Costa Rica.- Rev. Geol. Amér. Central, 19/20: 37-55.

SOTO, G \& ALVARADO, G.E., 2006: Eruptive history of Arenal Volcano, Costa Rica, 7 Ka to present.- Journal of Vulcanology and Geothermal Research, 157: 254-269.

VARGAS-CAMPOS, C.A., 2002: Diagnostico de los recursos hídricos subterráneos en la zona Guanacaste Norte.- 95 págs. SENARA, San José.

WOODS, R.D., 1968: Screening of surface waves in soils.- Jour. Soil Mech., 94 (4): 951-979. 